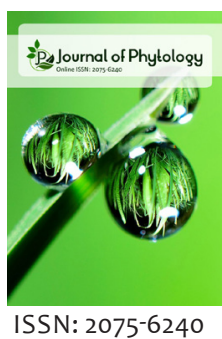

Received: April 01, 2021 Revised: June 11, 2021 Accepted: June 14, 2021 Published: June 28, 2021

*Corresponding authors: Jong Seok Park, E-mail: jongseok@cnu.ac.kr Sang Un Park, E-mail: supark@cnu.ac.kr

\section{Phenylalanine ammonia-lyase expression and pyranocoumarin accumulation in Angelica gigas plantlets exposed to light-emitting diodes}

\author{
Yeon Bok Kim, ${ }^{1, \dagger}$ Woo Tae Park', , Ramaraj Sathasivam², Seon Kyoung Yeo², \\ Gong In Lee ${ }^{3}$, Jong Seok Park ${ }^{4, *}$ and Sang Un Park ${ }^{2,5, *}$ \\ 'Department of Medicinal and Industrial Crops, Korea National College of Agriculture \& Fisheries, Jeonju, Korea, \\ ${ }^{2}$ Department of Crop Science, Chungnam National University, Daejeon, Korea, ${ }^{3}$ National Academy of Agricultural \\ Science, Rural Development Administration, Jeonju, Korea, ${ }^{4}$ Graduate school of Bio-Ai Convergence, Chungnam \\ National University, Daejeon, Korea, ${ }^{5}$ Department of Smart Agriculture Systems, Chungnam National University, \\ Daejeon, Korea \\ tContributed equally to this work
}

\begin{abstract}
Angelica gigas (Dang Gui) is an important medicinal plant. In this study, we examined the accumulation of pyranocoumarin (decursin and decursinol angelate) and the expression of phenylalanine ammonia-lyase (PAL) in Korean angelica plantlet grown under different light-emitting diodes (LEDs) (red, orange, green, blue, and white). Three weeks after LED exposure (WAE), the transcript levels of phenylalanine ammonia-lyase mRNA in seedlings grown under orange LEDs were 4-, 18-, and 7-fold higher than those in seedlings grown under green, blue, and white LEDs, respectively. The decursinol angelate content was almost double than the decursin content. The highest levels of decursin (3.2 mg/g dry weight) and decursinol angelate $(6 \mathrm{mg} / \mathrm{g}$ dry weight) were detected in plants grown under orange LEDs, at 2 WAE. Therefore, we suggest that orange LEDs may affect decursin and decursinol angelate accumulation. The findings of this study could help to determine an effective strategy for producing secondary metabolites in A. gigas using LED technology.
\end{abstract}

KEYWORDS: Angelica gigas, decursin, decursinol angelate, light-emitting diodes, phenylalanine ammonia-lyase, transcription

\section{INTRODUCTION}

Angelica gigas (Dang Gui), an important medicinal plant, has been widely used in traditional Korean medicine. This plant, known as Korean angelica, has dark purple flowers, whereas Chinese and Japanese angelica flowers (A. sinensis and A. acutiloba, respectively) are white (Ahn et al., 2008). (Lee et al., 2003a) and (Kim et al., 2008) reported that A. gigas roots have antioxidant activity, skin-whitening effects, and ultraviolet (UV)-protective effects. In addition, it is documented that A. sinensis has also been widely used in China for the remedy of several diseases, i.e. anemia, asthma, cardiovascular diseases, chronic bronchitis, hypertension, and rheumatic disorder (Lin et al., 1998; Lao et al., 2004; Lu et al., 2004). The major functional compounds in this plant are coumarins of decursin, decursinol, and nodakenin, and a variety of secondary metabolites are biosynthesized in A. sinensis. Additionally, decursin and decursinol angelate have been involved in antiandrogen receptor signaling, cytotoxicity, and neuroprotective activities (Konoshima et al., 1968; Pachaly et al., 1996; Lee et al., 2003b).

The quality and quantity of chemical compounds of medicinal plants are affected by climatic and edaphic factors such as temperature, duration of sun exposure, precipitation, soil, and exposure to light-emitting diodes (LEDs) (Kim et al., 2011; Tuan et al., 2013; Thwe et al., 2014). Among these factors, LEDs have many benefits for facilitating growth within regulated environments. These advantages comprise wavelength specificity, adjustable light intensity, high energy-conversion efficiency, longer life, and low thermal energy output (Okamoto et al., 1996; Schuerger et al., 1997). Red light can enhance starch content by reducing the translocation of photosynthates from

Copyright: $\odot$ The authors. This article is open access and licensed under the terms of the Creative Commons Attribution License (http://creativecommons.org/licenses/by/4.0/) which permits unrestricted, use, distribution and reproduction in any medium, or format for any purpose, even commercially provided the work is properly cited. Attribution - You must give appropriate credit, provide a link to the license, and indicate if changes were made. 
the leaves (Sæbø et al., 1995), whereas blue light is crucial for the development of chloroplast, chlorophyll synthesis, and stomata opening (Senger, 1982). Blue light has been shown to play a significant role in a broad range of plant processes, including photomorphogenesis, photosynthesis, phototropism, and stomatal opening (Staiger, 2008). Our group reported that red and blue lights cause substantial increases in catechin and rutin contents, respectively, in Fagopyrum tataricum sprouts (Thwe et al., 2014). The total carotenoid content of Tartary buckwheat sprouts grown under white LED was higher than that of sprouts grown under blue and red LEDs (Tuan et al., 2013).

Moreover, in a previous study from our laboratory, we reported that the expression level of genes involved in the anthocyanin biosynthetic pathway and anthocyanin accumulation in F. tataricum sprouts grown under light or dark conditions were significantly influenced (Li et al., 2012). The phenylpropanoid pathway plays a vital role in the production of secondary metabolic compounds in plants and acts as an intermediate for the production of various metabolites (Dixon and Paiva, 1995) (Figure 1). Phenylalanine ammonia-lyase (PAL) is one of the most key regulatory enzymes in the phenylpropanoid biosynthetic pathway which converts L-phenylalanine to trans-cinnamic acid by catalyzation process (Liu et al., 2006). In plants, the PAL gene has been extensively studied due to its significance in the production of numerous secondary metabolites. In A. gigas, PAL plays a vital role in the decursin and decursinol angelate biosynthetic pathway (Park et al., 2010).

\section{MATERIALS AND METHODS}

\section{Plant Materials and Growth Conditions}

A. gigas seeds were harvested in the fields of Youngju Agriculture Technical Center in March 2014. The seeds were washed for three days with tap water and transferred into pots filled with perlite-mixed soil. The seedlings were cultured in a growth chamber maintaining a flux rate of $120 \mu \mathrm{mol} \mathrm{m}^{-2} \mathrm{~s}^{-1}$, at $22-24^{\circ} \mathrm{C}$ and $70 \%$ humidity. After 5 weeks following germination, seedlings were exposed to different LEDs of red (SL5-RT501T ${ }^{-1}$, $625 \mathrm{~nm}$ ), orange (SL5-YT501T-1, $590 \mathrm{~nm})$, green (SL5SG501TZ, $525 \mathrm{~nm}$ ), blue (SL5-SB501T ${ }^{\mathrm{Z}}, 467 \mathrm{~nm}$ ), and white (SL5-SW501T ${ }^{-1}, 380 \mathrm{~nm}$ ) for 3weeks. LEDs light intensities were maintained at $72 \pm 3 \mu \mathrm{mol} \mathrm{m} \mathrm{m}^{-2}$. The seedlings were harvested at one, two, and three WAE. Harvested samples were immediately frozen in liquid nitrogen and then the samples were stored at $-80^{\circ} \mathrm{C}$.

RNA Extraction and Quantitative Real-Time Polymerase Chain Reaction (qRT-PCR)

RNA was extracted from each Korean angelica seedling using a Plant Total RNA Mini kit (Geneaid, Taiwan). For cDNA synthesis, the ReverTra Ace- $\alpha$ - Kit (Toyobo, Japan) was used according to the manufacturer's instructions. The cDNA was diluted 20 times with RNase-free water for qRT-PCR. Transcript levels of AgPAL and $\mathrm{AgC} 4 \mathrm{H}$ genes were analyzed by qRT-PCR (Bio-Rad, Hercules, CA) using a $2 \times$ Real-Time PCR Smart

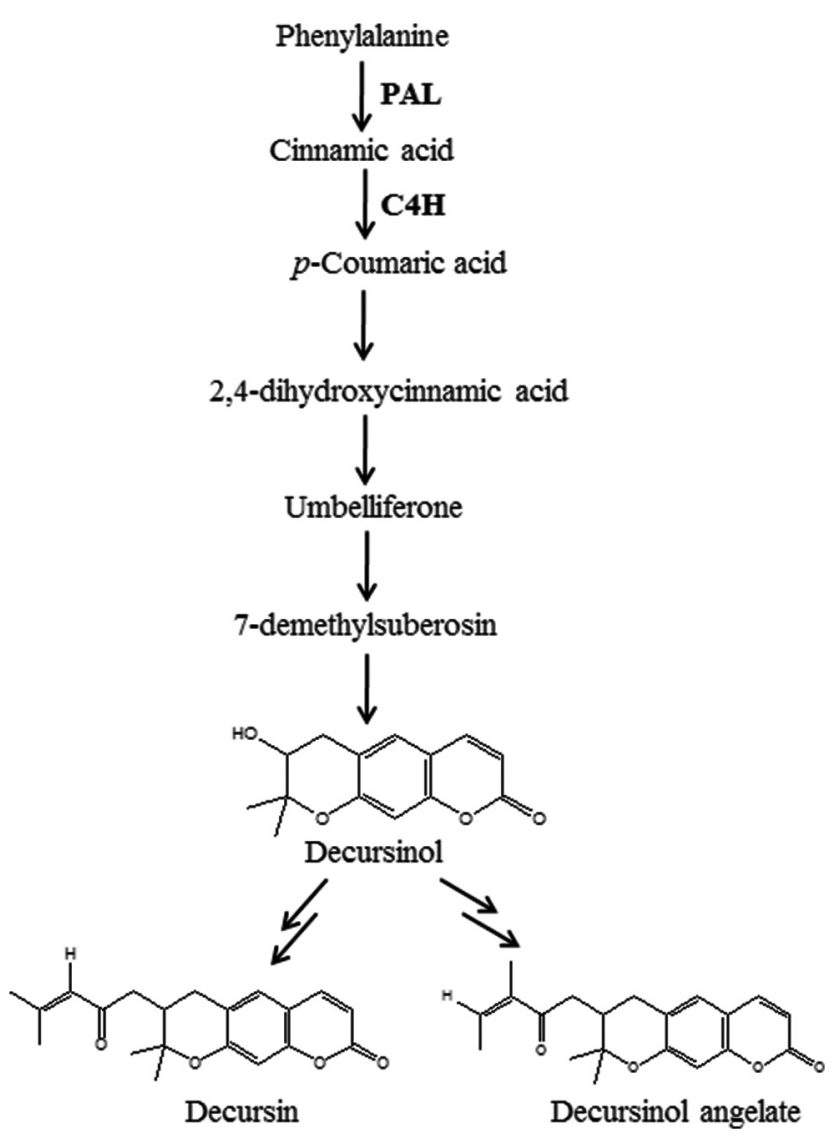

Figure 1: Schematic view of decursin and decursinol angelate biosynthetic pathway in Angelica gigas. PAL, phenylalanine ammonialyase; $\mathrm{C} 4 \mathrm{H}$, cinnamic acid 4-hydroxylase

mix (SolGent, Korea). Gene-specific primer sets were designed for qRT-PCR as follows: AgPAL- (forward: 5'-AAC AGC ACA ATC AAG ATG TGA ACT CC-3'; reverse: 5'-AAT TCT CCT CCA AAT GCC TCA AGT C-3') and Agl 18S- (forward: 5'-CTT AGT TGG TGG AGC GAT TTG TCT G-3'; reverse 5'-ACC TGT TAT TGC CTC AAA CTT CCG T-3’). qRT-PCR was conducted using a $2 \times$ Real-Time PCR Smart mix (SolGent, Korea). The qRT-PCR cyclic condition was as follows: $3 \mathrm{~min}$ at $95^{\circ} \mathrm{C}$ followed by 40 cycles of $10 \mathrm{~s}$ at $95^{\circ} \mathrm{C}, 10 \mathrm{~s}$ at $55^{\circ} \mathrm{C} \mathrm{s}$, and $30 \mathrm{~s}$ at $72^{\circ} \mathrm{C}$. All the reactions were carried out in triplicates. The housekeeping gene $18 \mathrm{~S}$ ribosomal gene (Accession number DQ647697) was used as an internal control.

\section{High-Performance Liquid Chromatography (HPLC) Analysis}

Extraction and analysis of decursin and decursinol angelate was analyzed based on the protocol described by Park et al. (Park et al., 2010) with slight modification. Dried $(500 \mathrm{mg}$ ) fine powder samples were mixed with $70 \%$ ethanol $(30 \mathrm{~mL})$ and incubated for $1 \mathrm{~h}$ in a water bath at $50{ }^{\circ} \mathrm{C}$. The mixture was centrifuged at 10,000 rpm for $15 \mathrm{~min}$. Twenty-five milliliter of supernatant was concentrated by using a vacuum concentrator and then extracted with dichloromethane three times. Then this mixture was dried under vacuum, and add $1 \mathrm{ml}$ acetonitrile. These mixtures were passed through a $0.45 \mu \mathrm{m}$ PTFE filter. The separation of compounds was carried out using a Futecs 
model of NS-4000 HPLC system (Daejeon, Korea) equipped with a reversed-phase OptimaPak Cl8 $(5 \mu \mathrm{m}, 250 \mathrm{~mm} \times$ $4.6 \mathrm{~mm}$ ) column and UV detector. The sample injection volume was $10 \mu \mathrm{L}$. The mobile phase used in this system was $40 \%$ acetonitrile, $50 \%$ water, and $10 \%$ tetrahydrofuran. The flow rate of the solvent was $0.8 \mathrm{~mL} / \mathrm{min}$ and the column temperature was $35^{\circ} \mathrm{C}$, and the chromatogram was acquired at $280 \mathrm{~nm}$. Decursin and decursinol angelates were identified based on their HPLC peak area ratios and quantified based on the retention time, peak areas, and response factor with the respective external standards.

\section{Statistical Analysis}

All data were evaluated using Statistical Analysis System (SAS version 9.2, Cary, NC, USA). The mean and standard deviation were calculated from three biological replicates. The significant differences among the means were calculated by using Duncan Multiple Range Test.

\section{RESULTS AND DISCUSSION}

In this study, pyranocoumarin (decursin and decursinol angelate) accumulation and Phenylalanine ammonia-lyase (PAL) expression were analyzed in Korean angelica seedlings grown under different LED lights. The study aimed to examine the growth response of Korean angelica seedlings under different LED lights (red, blue, orange, green, and white). In addition, for better understanding, the regulation of genes engaged in the phenylpropanoid biosynthetic pathway, especially of PAL. Moreover, we had analyzed the pyranocoumarin accumulation in A. gigas plantlets in response to LEDs.
A. gigas seedlings were grown under different light conditions, i.e. white, blue, orange, green, and red LEDs for three days and the changes in transcript levels of AgPAL were measured at 1-week intervals using qRT-PCR (Figure 2). The gene expression levels in seedlings grown under green, blue, and white lights were almost similar, whereas those of seedlings grown under red and orange LEDs were dissimilar. Specifically, AgPAL transcript levels in plants grown under red and orange LEDs were the highest at 3 WAE, whereas they were highest in plants irradiated under green, blue, and white LEDs at 2 WAE. Transcript levels gradually increased from 1 to 3 WAE in plants grown under orange LEDs. The expression levels of AgPAL in seedlings grown under orange LEDs were 4-, 18-, and 7-fold higher than those in seedlings raised under green, blue, and white LEDs, respectively, at 3 WAE. Moreover, the transcript levels of AgPAL in plants grown under orange LEDs were 70- and 2-fold higher at 3 WAE than at 1 and 2 WAE, respectively. For plants grown under red LEDs, AgPAL expression levels were 9-fold greater at 2 and 3 WAE than at 1 WAE. Finally, for plants grown under green LEDs, AgPAL expression levels were 10- and 2-fold higher at 2 WAE than at 1 and 3 WAE, respectively. Recently, our group reported that the expression of most genes involved in flavonoid biosynthesis was observed on day two after LED exposure, particularly for FtPAL andFtF3'H, showing greater expression in Tartary buckwheat sprouts grown under blue and white than in those grown under red LED (Thwe et al., 2014).

Most of the carotenoid biosynthetic pathway genes in Tartary buckwheat sprouts grown under white LEDs expressed higher transcript levels at day eight after sowing compared with gene expression in plants raised under blue and red LEDs conditions (Tuan et al., 2013). However, in this study, the expression level

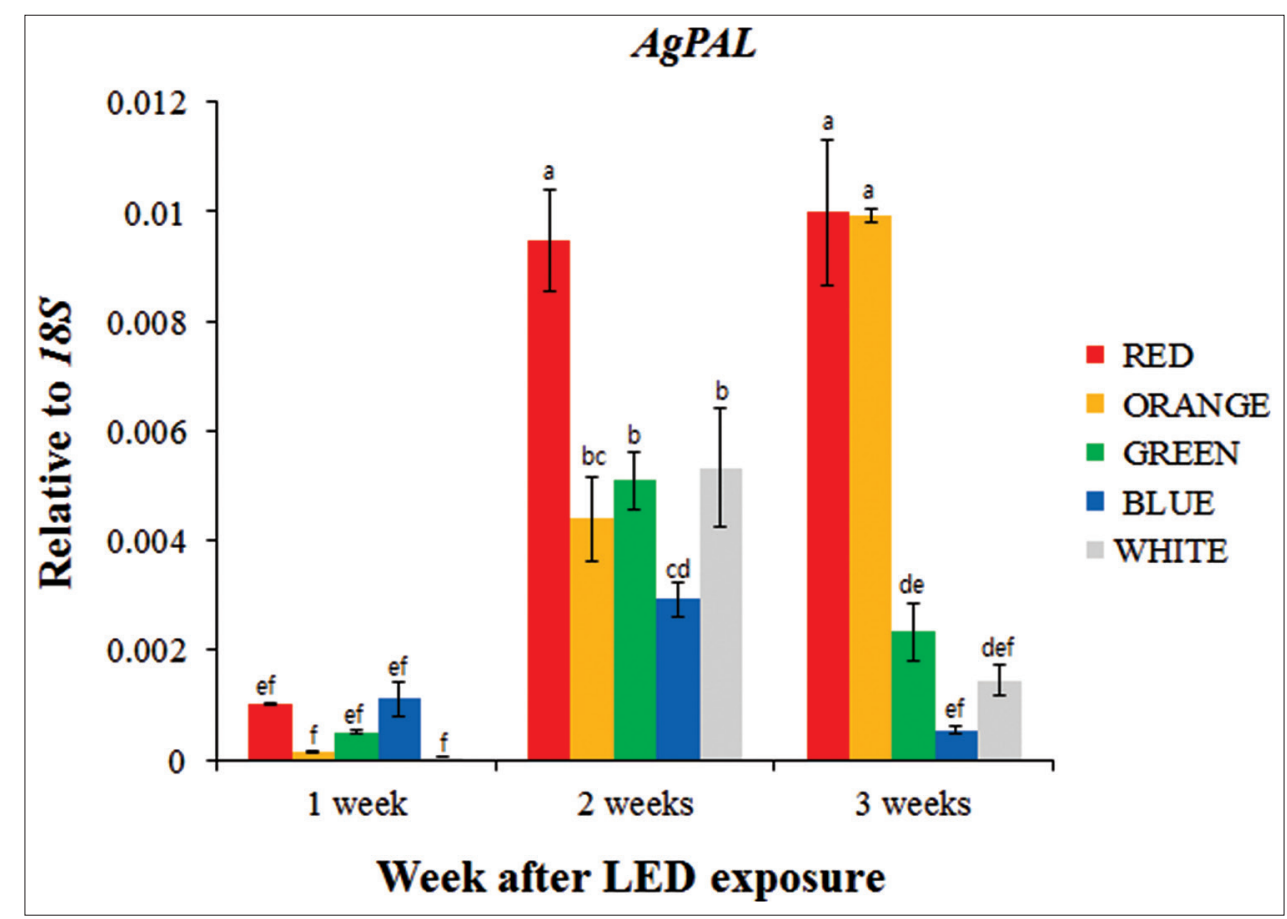

Figure 2: Variation of transcript levels of AgPAL in seedlings grown under LED lights in A. gigas. The values of transcript levels were determined using three biological replicates and analyzed relative to those of $18 \mathrm{~S}$ 
of AgPAL in the seedlings of Tartary buckwheat under red LEDs was greater than that of seedlings grown under blue and white lights. Thus, our data proposed that the expression level of AgPAL was dependent on the plant and the LED condition. Martín-Cabrejas et al., 2003 found that light can induce metabolic changes during germination. In addition, Archetti et al., 2009 and Karageorgou et al., 2008 reported that metabolite changes under light conditions may occur because of the accumulation of anthocyanins, which are vacuolar flavonoids, and that anthocyanin content is positively correlated with phenolics in several species.

Previously, we reported that the transcription of both AgPAL and $\mathrm{AgC} 4 \mathrm{H}$ has highly enhanced with $300 \mu \mathrm{M}$ methyl jasmonate for 6 or $12 \mathrm{~h}$, respectively, and that these transcripts were highly expressed in the A. gigas roots (Park et al., 2010). In addition, the transcript levels of $\mathrm{AgPAL}$ and $\mathrm{AgC} 4 \mathrm{H}$ showed a similar pattern. However, in the current study, transcription patterns of AgPAL varied. Unlike AgPAL showed very low transcription compared with Agl8S (data not shown). Finally, previous studies have primarily used only red, blue, and white LEDs. In the current study, we used green and orange LEDs in addition to red, blue, and white LEDs. Interestingly, unlike the results of other studies, we also found that red and orange LEDs had substantial effects on the transcription of AgPAL.

Next, we analyzed the accumulation of decursin and decursinol angelates during A. gigas seedling development by HPLC (Figure 3). The decursinol angelate content was two-fold higher

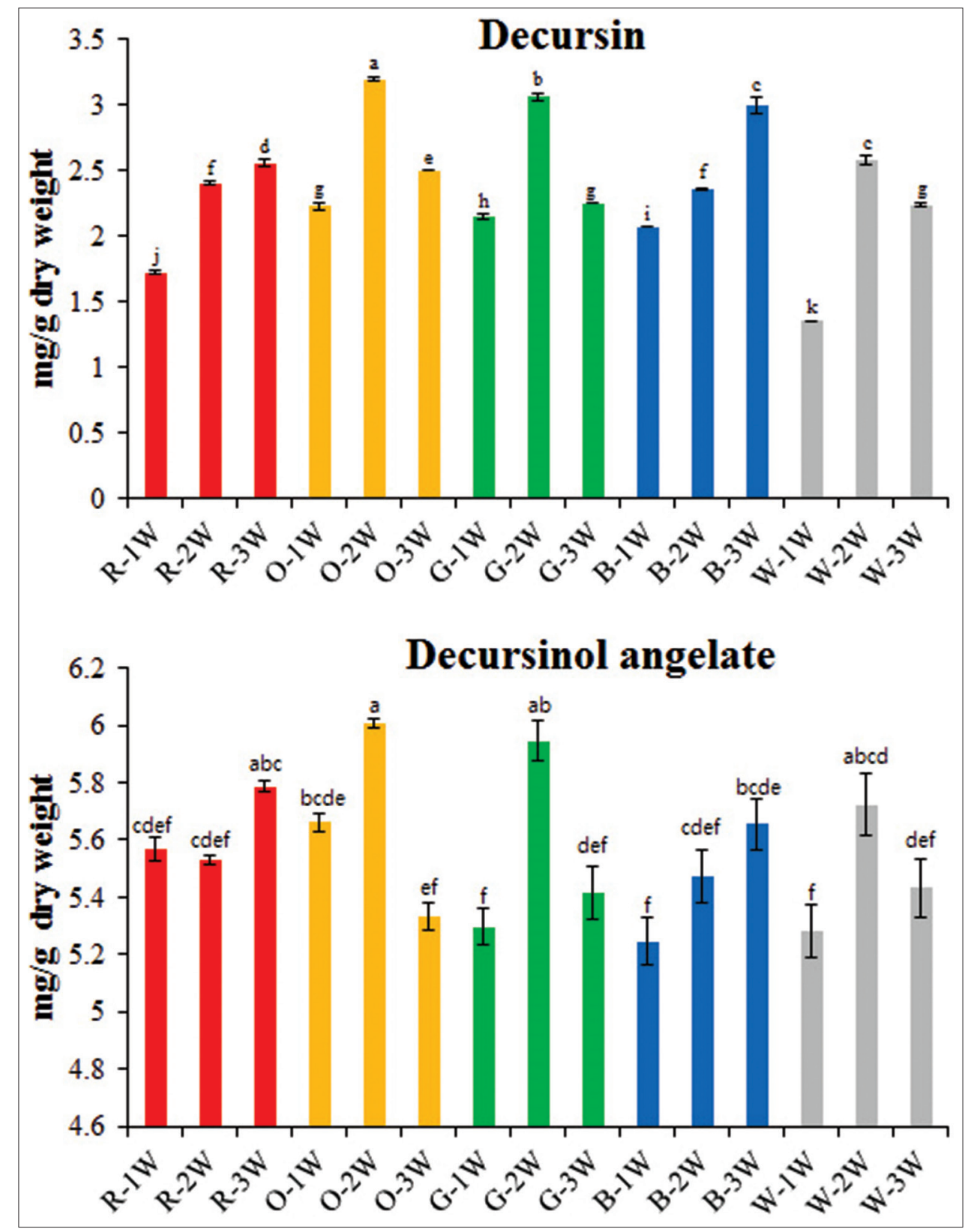

Figure 3: Analysis of decursin and decursinol angelate in seedlings grown under LED lights in $A$. gigas. The symbols $\mathrm{R}, \mathrm{O}, \mathrm{G}, \mathrm{B}$, and W represent red, orange, green, blue, and white, respectively; 1W, 1 week; $2 \mathrm{~W}, 2$ weeks; 3W, 3 weeks 
than that of decursin content. Additionally, there was a significant difference in decursin and decursinol angelate accumulation at different sampling times. The highest decursin content was detected in plants grown under orange LEDs at 2 WAE (3.2 mg/g DW), whereas the lowest decursin content was observed in plants grown under white LEDs at 1 WAE (1.4 mg/g DW). Interestingly, AgPAL showed the highest expression in seedlings grown under orange LEDs at 3 WAE. The decursin content of seedlings grown under red and blue LEDs gradually increased from 1 to 3 WAE, whereas that in seedlings grown under orange, green, and white LEDs was the highest at 2 WAE.

In general, the light changes the intensity of the pigment by interceding the expression of genes involved in the anthocyanin biosynthetic pathway (Sheoran et al., 2006). Anthocyanin, flavonols, and phenolic acids are induced rapidly by irradiation, whereas dihydrochalcones, flavanols, and procyanidins do not vary in mature versus ripe apple fruits (Bakhshi and Arakawa, 2006). It is reported that the epicatechin content of T8 Tartary buckwheat was affected by light, suggesting that the effect of light on catechin and epicatechin content may depend on the cultivar of Tartary buckwheat (Kim et al., 2014). In addition, Thwe et al., 2014 found that LEDs and fluorescent lights have comparable effects on rutin content in sprouts of Tartary buckwheat. Blue light increases the accumulation of anthocyanins, which play a significant role in antioxidant activity (Duan et al., 2007).

\section{CONCLUSION}

In the present study, decursin and decursinol angelate content were the highest under orange LEDs. Consistent with this, AgPAL mRNA levels were highest in plants grown under orange LEDs at 3 WAE. Therefore, we suggest that orange LEDs may affect decursin and decursinol angelate accumulation through PAL. Furthermore, we speculate that the findings of this study will help to establish an effective procedure for the accumulation of secondary metabolites in A. gigas using LED technology.

\section{ACKNOWLEDGMENTS}

This work was supported by Institute of Information \& communications Technology Planning \& Evaluation (IITP) grant funded by the Korea government (MSIT) (No.2020-001441, Artificial Intelligence Convergence Research Center (Chungnam National University)).

\section{AUTHOR'S CONTRIBUTIONS}

J.S.P. and S.U.P. designed the experiments and analyzed the data. Y.B.K., W.T.P., R.S., S.K.Y., G.I.L., performed the experiments and analyzed the data. Y.B.K., J.S.P., and S.U.P. wrote the manuscript. All authors read and approved the final manuscript.

\section{REFERENCES}

Ahn, M. -J., Lee, M. K., Kim, Y. C., \& Sung, S. H. (2008). The simultaneous determination of coumarins in Angelica gigas root by high performance liquid chromatography-diode array detector coupled with electrospray ionization/mass spectrometry. Journal of Pharmaceutical and Biomedical Analysis, 46(2), 258-266. https://doi. org/10.1016/j.jpba.2007.09.020

Archetti, M., Döring, T. F., Hagen, S. B., Hughes, N. M., Leather, S. R., Lee, D. W., Lev-Yadun, S., Manetas, Y., Ougham, H. J., \& Schaberg, P. G. (2009). Unravelling the evolution of autumn colours: an interdisciplinary approach. Trends in Ecology and Evolution, 24(3), 166-173. https:// doi.org/10.1016/j.tree.2008.10.006

Bakhshi, D., \& Arakawa, O. (2006). Induction of phenolic compounds biosynthesis with light irradiation in the flesh of red and yellow apples. Journal of Applied Horticulture, 8(2), 101-104.

Dixon, R. A., \& Paiva, N. L. (1995). Stress-induced phenylpropanoid metabolism. The Plant Cell, 7(7), 1085. https://doi.org/10.1105/ tpc.7.7.1085

Duan, X., Jiang, Y., Su, X., Zhang, Z., \& Shi, J. (2007). Antioxidant properties of anthocyanins extracted from litchi (Litchi chinenesis Sonn.) fruit pericarp tissues in relation to their role in the pericarp browning. Food Chemistry, 101(4), 1365-1371. https://doi.org/10.1016/j. foodchem.2005.06.057

Karageorgou, P., Buschmann, C., \& Manetas, Y. (2008). Red leaf color as a warning signal against insect herbivory: honest or mimetic? Flora-Morphology, Distribution, Functional Ecology of Plants, 203(8), 648-652. https://doi.org/10.1016/j.flora.2007.10.006

Kim, C. -H., Kwon, M. -C., Han, J. -G., Na, C. -S., Kwak, H. -G., Choi, G. -P., Park, U. -Y., \& Lee, H. -Y. (2008). Skin-whitening and UV-protective effects of Angelica gigas Nakai extracts on ultra high pressure extraction process. Korean Journal of Medicinal Crop Science, 16(4), 255-260.

Kim, E. J., Kwon, J., Park, S. H., Park, C., Seo, Y. -B., Shin, H. -K., Kim, H. K., Lee, K. -S., Choi, S. -Y., \& Ryu, D. H. (2011). Metabolite profiling of Angelica gigas from different geographical origins using ${ }^{1} \mathrm{H}$ NMR and UPLC-MS analyses. Journal of Agricultural and Food Chemistry, 59(16), 8806-8815. https://doi.org/10.1021/jf2016286

Kim, Y. B., Thwe, A. A., Kim, Y., Li, X., Cho, J. W., Park, P. B., Valan Arasu, M., Abdullah Al-Dhabi, N., Kim, S. -J., \& Suzuki, T. (2014). Transcripts of anthocyanidin reductase and leucoanthocyanidin reductase and measurement of catechin and epicatechin in tartary buckwheat. The Scientific World Journal, Article ID 726567. https:// doi.org/10.1155/2014/726567

Konoshima, M., Chi, H. J., \& Hata, K. (1968). Coumarins from the root of Angelica gigas Nakai. Chemical and Pharmaceutical Bulletin, 16(6), 1139-1140. https://doi.org/10.1248/cpb.16.1139

Lao, S., Li, S., Kan, K. K., Li, P., Wan, J., Wang, Y., Dong, T. T., \& Tsim, K. W. (2004). Identification and quantification of 13 components in Angelica sinensis (Danggui) by gas chromatography-mass spectrometry coupled with pressurized liquid extraction. Analytica Chimica Acta, 526(2), 131-137. https://doi.org/10.1016/j.aca.2004.09.050

Lee, J. -H., Chae, H. -J., Kim, D. -H., Lee, S. -H., Park, S. -Y., \& Kang, Y. -G. (2003b). HPLC analysis and extraction methods of decursin and decursinol angelate in Angelica gigas roots. Korean Journal of Pharmacognosy, 34(3), 201-205.

Lee, S. -H., Lee, Y. -S., Jung, S. -H., Shin, K. -H., Kim, B. -K., \& Kang, S. -S. (2003a). Antioxidant activities of decursinol angelate and decursin from Angelica gigas roots. Natural Product Sciences, 9(3), 170-173.

Li, X., Thwe, A. A., Park, N. I., Suzuki, T., Kim, S. J., \& Park, S. U. (2012). Accumulation of phenylpropanoids and correlated gene expression during the development of tartary buckwheat sprouts. Journal of Agricultural and Food Chemistry, 60(22), 5629-5635. https://doi. org/10.1021/jf301449a

Lin, L. -Z., He, X. -G., Lian, L. -Z., King, W., \& Elliott, J. (1998). Liquid chromatographic-electrospray mass spectrometric study of the phthalides of Angelica sinensis and chemical changes of Z-ligustilide. Journal of Chromatography A, 810(1-2), 71-79. https:// doi.org/10.1016/S0021-9673(98)00201-5

Liu, R., Xu, S., Li, J., Hu, Y., \& Lin, Z. (2006). Expression profile of a PAL gene from Astragalus membranaceus var. Mongholicus and its crucial role in flux into flavonoid biosynthesis. Plant Cel/ Reports, 25(7), 705-710. https://doi.org/10.1007/s00299-005-0072-7

Lu, G. -H., Chan, K., Chan, C. -L., Leung, K., Jiang, Z. -H., \& Zhao, Z. -Z. (2004). Quantification of ligustilides in the roots of Angelica sinensis and related umbelliferous medicinal plants by high-performance liquid chromatography and liquid chromatography-mass spectrometry. Journal of Chromatography A, 1046(1-2), 101-107. https://doi. org/10.1016/j.chroma.2004.06.083 
Martín-Cabrejas, M. A., Ariza, N., Esteban, R., Mollá, E., Waldron, K., \& López-Andréu, F. J. (2003). Effect of germination on the carbohydrate composition of the dietary fiber of peas (Pisum sativum L.). Journal of Agricultural and Food Chemistry, 51(5), 1254-1259. https://doi. org/10.1021/jf0207631

Okamoto, K., Yanagi, T., \& Kondo, S. (1996). Growth and morphogenesis of lettuce seedlings raised under different combinations of red and blue light. II Workshop on Environmental Regulation of Plant Morphogenesis 435, pp. 149-158. https://doi.org/10.17660/ ActaHortic. 1997.435.14

Pachaly, P., Treitner, A., \& Sin, K. S. (1996). New coumarin glycosides from Angelica gigas roots. Pharmazie, 51, 57-61.

Park, J. H., Park, N. I., Xu, H., \& Park, S. U. (2010). Cloning and characterization of phenylalanine ammonia-lyase and cinnamate 4-hydroxylase and pyranocoumarin biosynthesis in Angelica gigas. Journal of Natural Products, 73(8), 1394-1397. https://doi.org/10.1021/np1003356

Sæbø, A., Krekling, T., \& Appelgren, M. (1995). Light quality affects photosynthesis and leaf anatomy of birch plantlets in vitro. Plant Cell, Tissue and Organ Culture, 41, 177-185. https://doi.org/10.1007/ BF00051588

Schuerger, A. C., Brown, C. S., \& Stryjewski, E. C. (1997). Anatomical features of pepper plants (Capsicum annuum L.) grown under red light-emitting diodes supplemented with blue or far-red light. Annals of Botany, 79(3), 273-282. https://doi.org/1006/anbo.1996.0341

Senger, H. (1982). The effect of blue light on plants and microorganisms. Photochemistry and Photobiology, 35(6), 911-920. https://doi. org/10.1111/j.1751-1097.1982.tb02668.x

Sheoran, I. S., Dumonceaux, T., Datla, R., \& Sawhney, V. K. (2006). Anthocyanin accumulation in the hypocotyl of an ABA-over producing male-sterile tomato (Lycopersicon esculentum) mutant. Physiologia Plantarum, 127(4), 681-689. https://doi.org/10.1111/j.13993054.2006.00697.x

Staiger, D. (2008). Light and plant development. Annual Plant Reviews. Annals of Botany, 101(3), 480-481.

Thwe, A. A., Kim, Y. B., Li, X., Seo, J. M., Kim, S. -J., Suzuki, T., Chung, S. -O., \& Park, S. U. (2014). Effects of light-emitting diodes on expression of phenylpropanoid biosynthetic genes and accumulation of phenylpropanoids in Fagopyrum tataricum sprouts. Journal of Agricultural and Food Chemistry, 62(21), 4839-4845. https://doi. org/10.1021/jf501335q

Tuan, P. A., Thwe, A. A., Kim, Y. B., Kim, J. K., Kim, S. -J., Lee, S. Chung, S. -O., \& Park, S. U. (2013). Effects of white, blue, and red light-emitting diodes on carotenoid biosynthetic gene expression levels and carotenoid accumulation in sprouts of tartary buckwheat (Fagopyrum tataricum Gaertn.). Journal of Agricultural and Food Chemistry, 61(50), 12356-12361. https://doi.org/10.1021/jf4039937 